\title{
Mass Spectrum of Dirac Equation with Local Parabolic Potential
}

\author{
Yingqiu Gu \\ School of Mathematical Science, Fudan University, Shanghai, China \\ Email: yqgu@fudan.edu.cn
}

How to cite this paper: Gu, Y.Q. (2020)

Mass Spectrum of Dirac Equation with Local Parabolic Potential. Journal of High Energy Physics, Gravitation and Cosmolo$g y, 6,20-33$.

https://doi.org/10.4236/jhepgc.2020.61003

Received: August 20, 2019

Accepted: November 24, 2019

Published: November 27, 2019

Copyright $\odot 2020$ by author(s) and Scientific Research Publishing Inc. This work is licensed under the Creative Commons Attribution International License (CC BY 4.0).

http://creativecommons.org/licenses/by/4.0/

(c) (i) Open Access

\begin{abstract}
In this paper, we solve the eigen solutions to the Dirac equation with local parabolic potential which is approximately equal to the short distance potential generated by spinor itself. The energy spectrum is quite different from that with Coulomb potential. The mass spectrum of some baryons is similar to this one. The angular momentum-mass relation is quite similar to the Regge trajectories. The parabolic potential has a structure of asymptotic freedom near the center and confinement at a large distance. So, the results imply that, the local parabolic potential may be more suitable for describing the nuclear potential. The procedure of solving can also be used for some other cases of Dirac equation with complicated potential.
\end{abstract}

\section{Keywords}

Regge Trajectory, Parabolic Potential, Mass Spectrum, Baryon, Short Distance Potential

\section{Introduction}

For hadrons, the relation between mass $m$ and quantum numbers $(n, J)$ is usually described by the Regge-Chew-Frautschi formula [1] [2],

$$
m^{2}=a n+b J+m_{0}
$$

where $\left(a, b, m_{0}\right)$ are constants for the exited states of the same kind particle. In many cases, the coefficients satisfy $b \leq a \leq 2 b$ [3] [4]. The Regge trajectory is an important tool widely used to analyze the spectroscopy of mesons and baryons. Various theoretical models have been constructed to explain the mass spectra of particles and to derive the Regge trajectories, such as non-relativistic quark models [5]-[13], flux tube model or similar string model [3] [14]-[22], semi-relativistic potential model [23] [24] [25] [26], relativistic potential model [27] [28] [29] [30] 
[31], quantum chromodynamics (QCD) sum rule [32] [33] [34], color hyperfine interaction [35] [36] [37] [38], Lattice QCD model [39] [40] [41] [42] [43] and so on. There are also some Regge phenomenology investigations [3] [21] [44]-[48]. By statistical and regressive method to get the relation $m=f(n, J)$.

In many models, the total potential between quarks is given by Cornell potential with some hyperfine terms of correction, and the mass spectrum is solved in relative Jacobi coordinates [8] [10] [13] [30] [31] [35] [49]. In [49], by semi classical approximation and Bohr-Sommerfeld quantization, the Regge-like relation $E \sim L^{2 \alpha /(\alpha+2)}$ and $E \sim n^{2 \alpha /(\alpha+2)}$ for large $(n, L)$ is derived for power-law confining potentials $V \propto r^{\alpha}$. By the phenomenological researches, we also find that the Regge-Chew-Frautschi formula (1.1) is only approximately valid, and a little nonlinearity always exists [44] [45] [47]. The specific Regge trajectories depend on concrete confining potential. However, no matter what confining potential is, the analytic relation $m=f(n, J)$ for the excited states always exists.

Recently, a number of experimental data for highly exited resonances were reported [50]-[58]. These data provide opportunity to check the previous calculations and develop more effective models. As pointed out in [58], a better understanding of the nucleon as a bound state of quarks and gluons as well as the spectrum and internal structure of excited baryons remains a fundamental challenge and goal in hadronic physics. In particular, the mapping of the nucleon excitations provides access to strong interactions in the domain of quark confinement. While the peculiar phenomenon of confinement is experimentally well established and believed to be true, it remains analytically unproven and the connection to quantum chromodynamics (QCD) - the fundamental theory of the strong interactions-is only poorly understood. In the early years of the 20th century, the study of the hydrogen spectrum has established without question that the understanding of the structure of a bound state and of its excitation spectrum needs to be addressed simultaneously. The spectroscopy of excited baryon resonances and the study of their properties are thus complementary to understanding the structure of the nucleon in deep inelastic scattering experiments that provide access to the properties of its constituents in the ground state.

The quark models employ multiplets of spinors and nonlinear interactive vectors with gauge symmetries, which are too complicated to get exact solutions and an overview for the properties. In this paper we examine the following simple and closed Dirac equation with short range self-generating vector potential $\Phi_{\mu}$,

$$
\mathcal{L}=\phi^{+} \alpha^{\mu}\left(i \partial_{\mu}-s \Phi_{\mu}\right) \phi-\mu c \breve{\gamma}+\frac{1}{2}\left(\partial_{\mu} \Phi_{\alpha} \partial^{\mu} \Phi^{\alpha}-b^{2} \Phi_{\mu} \Phi^{\mu}\right),
$$

in which $\breve{\gamma}=\phi^{+} \gamma \phi$. (1.2) has plentiful spectra. By the Regge trajectories we find the excited states may be relevant to some of baryons.

\section{Equations and Simplification}

At first, we introduce some notations. Denote the Minkowski metric by $\eta_{\mu v}=\operatorname{diag}(1,-1,-1,-1)$, Pauli matrices by 


$$
\vec{\sigma}=\left(\sigma^{j}\right)=\left\{\left(\begin{array}{ll}
0 & 1 \\
1 & 0
\end{array}\right),\left(\begin{array}{cc}
0 & -i \\
i & 0
\end{array}\right),\left(\begin{array}{cc}
1 & 0 \\
0 & -1
\end{array}\right)\right\} .
$$

Define $4 \times 4$ Hermitian matrices as follows

$$
\alpha^{\mu}=\left\{\left(\begin{array}{ll}
I & 0 \\
0 & I
\end{array}\right),\left(\begin{array}{ll}
0 & \vec{\sigma} \\
\vec{\sigma} & 0
\end{array}\right)\right\}, \quad \gamma=\left(\begin{array}{cc}
I & 0 \\
0 & -I
\end{array}\right), \quad \beta=\left(\begin{array}{cc}
0 & -i I \\
i I & 0
\end{array}\right)
$$

where $\mu \in\{0,1,2,3\}, \quad x^{0}=c t$ and $\alpha^{\mu}=\gamma^{0} \gamma^{\mu}$. By variation of (1.2) we get the Dirac equation and dynamics of $\Phi^{\mu}$,

$$
\begin{gathered}
\alpha^{\mu}\left(\hbar i \partial_{\mu}-s \Phi_{\mu}\right) \phi=\mu c \gamma \phi . \\
\partial_{\alpha} \partial^{\alpha} \Phi^{\mu}+b^{2} \Phi^{\mu}=-s \breve{\alpha}^{\mu}, \quad \breve{\alpha}^{\mu}=\phi^{+} \alpha^{\mu} \phi .
\end{gathered}
$$

For the eigen states of $\phi$, only the magnetic quantum number $m_{z}$ and the sipn $s$ are conserved. So the eigen solution takes the following form

$$
\phi=\left(u_{1}, u_{2} \mathrm{e}^{\varphi i},-i v_{1},-i v_{2} \mathrm{e}^{\varphi i}\right)^{\mathrm{T}} \exp \left(m_{z} \varphi i-\frac{m c^{2}}{\hbar} i t\right),
$$

where the index "T" stands for transpose, $m_{z} \in\{0, \pm 1, \pm 2, \cdots\}$, and $u_{k}, v_{k}(k=1,2)$ are real functions of $r$ and $\theta$. However, the exact solution of (2.5) does not exist, and we have to solve it by effective algorithm [59] [60]. Since the numerical solutions are also unhelpful to understand the global structure of the mass spectrum, we seek for the approximate analytic solutions in this paper.

Different from the case of an electron, a proton has a hard core with charge distribution, and the radius of the distribution is about $1 \times 10^{-15} \mathrm{~m}$. The following calculation shows the local parabolic potential is approximately equal to $\Phi_{\mu}$ near the center, then we have

$$
\Phi_{0} \doteq\left(\frac{w^{2} r^{2}}{2 \rho^{2}}-2(1-\eta)\right) \mu c, \quad \vec{\Phi} \doteq 0, \quad(r<12 \rho),
$$

in which $w$ is the strength factor, $\eta$ is a parameter to adjust the depth of confinement to fit the true confining potential. $\rho=\frac{\hbar}{\mu c}$ is the theoretical Compton wave length, which is used for nondimensionalization of the Dirac equation.

In order to simplify (1.2), we make transformation [60]

$$
g=u_{1}+u_{2} i, \quad f=v_{1}-v_{2} i .
$$

Substituting (2.5), (2.6) and (2.7) into (1.2) we get Lagrangian as

$$
\mathcal{L}=\left(\mathcal{L}_{0}+\mathcal{L}_{f}\right) \mu c
$$

in which we defined

$$
\begin{aligned}
\mathcal{L}_{0} \equiv & \Re\left\langle\mathrm{e}^{\theta i}\left(-\bar{g}\left(\partial_{r}+\frac{i}{r} \partial_{\theta}\right) f+f\left(\partial_{r}+\frac{i}{r} \partial_{\theta}\right) \bar{g}\right)\right\rangle \rho \\
& -\frac{i}{r \sin \theta}\left(m_{z}+\frac{1}{2}\right)(\bar{g} \bar{f}-g f) \rho+\varepsilon\left(|g|^{2}+|f|^{2}\right) \\
& +\left(\frac{w^{2} r^{2}}{2 \rho^{2}}-2(1-\eta)\right)|g|^{2}-(2+\kappa)|f|^{2},
\end{aligned}
$$


where $\mathfrak{R}\langle\rangle$ stands for taking real part, and

$$
\mathcal{L}_{f} \equiv\left(\kappa+\frac{w^{2} r^{2}}{2 \rho^{2}}-2(1-\eta)\right)|f|^{2} .
$$

In (2.9), $\varepsilon$ is relative mass defect defined by

$$
m c^{2}=(1-\varepsilon) \mu c^{2}, \quad \varepsilon=\frac{\mu-m}{\mu},
$$

and $\rho$ is used as length unit, $\kappa$ is a constant to let $\left|\int_{0}^{\infty} \mathcal{L}_{f} r^{2} \mathrm{~d} r\right| \rightarrow 0$ so that convergent rate of the procedure is optimized. In the case (2.6), we set $\kappa=(1-\eta)$ which is about the mean value of the potential in the effective domain, and then $\mathcal{L}_{f}$ can be omitted for the 0 th order approximation. For proton we have

$$
\rho=\frac{\hbar}{\mu c}=(1-\varepsilon) \frac{\hbar}{m_{p} c}, \lambda=\frac{\hbar}{m_{p} c}=2.1037 \times 10^{-16} \mathrm{~m} .
$$

In (2.8), $\mathcal{L}_{0}$ almost keeps all invariance of relativity and has simple and complete eigensolutions, which can be used as the bases of Hilbert space of representation. $\mathcal{L}_{f}$ is the trouble terms with small energy, which acts as perturbation in the calculation. If taking $\mu c=1, \rho=1,(2.8)$ becomes dimensionless.

For (2.9), the rigorous eigen solutions take the following form [60]

$$
g=U(r)[P(\theta)+Q(\theta) i], \quad f=V(r)[P(\theta)+Q(\theta) i] \mathrm{e}^{-i \theta} .
$$

By variation of (2.9), we get

$$
\begin{gathered}
\partial_{\theta} P=\cot \theta m_{z} P+\left(m_{z}+K\right) Q, \\
\partial_{\theta} Q=-\cot \theta\left(m_{z}+1\right) Q+\left(m_{z}+1-K\right) P,
\end{gathered}
$$

in which $K= \pm 1, \pm 2, \cdots$ corresponding to orbital angular momentum, $P, Q$ are associated Legendre functions. The radial functions satisfy

$$
\begin{gathered}
\partial_{r}^{2} U+\frac{2}{r} \partial_{r} U-\left(\frac{K(K-1)}{r^{2}}-\frac{2 \mathcal{E}}{\rho^{2}}+\frac{(3-\varepsilon-\eta) w^{2} r^{2}}{2 \rho^{4}}\right) U=0, \\
V=\frac{\left(r \partial_{r} U-(K-1) U\right) \rho}{(3-\varepsilon-\eta) r},
\end{gathered}
$$

in which we defined

$$
\begin{aligned}
& \mathcal{E}=\frac{1}{2}(2-\varepsilon-2 \eta)(3-\varepsilon-\eta) \\
&= \frac{1}{2}\left((1-2 \eta)(2-\eta)+3(1-\eta) M+M^{2}\right),
\end{aligned}
$$

Or inversely,

$$
\varepsilon=\frac{1}{2}\left(5-3 \eta-\sqrt{(1+\eta)^{2}+8 \varepsilon}\right),
$$

where $M=\frac{m}{\mu}$ is dimensionless mass. The above equations can be easily solved, and the solutions are all elementary functions. The normalizing conditions are as follows 


$$
\int_{0}^{\pi}\left(P^{2}+Q^{2}\right) 2 \pi \sin \theta \mathrm{d} \theta=1, \quad \int_{0}^{\infty}\left(U^{2}+V^{2}\right) r^{2} \mathrm{~d} r=1 .
$$

\section{Eigen Solutions to the Equation}

For (2.16), we have the solution

$$
\begin{gathered}
U=\left(C_{1} r^{K-1}+C_{2} r^{-K}\right) L_{n-1}^{J}\left(\frac{2 r^{2}}{r_{n}^{2}}\right) \exp \left(-\frac{r^{2}}{r_{n}^{2}}\right), \\
n=1,2,3, \cdots, \quad J=\left|K-\frac{1}{2}\right|=\frac{1}{2}, \frac{3}{2}, \frac{5}{2}, \cdots,
\end{gathered}
$$

where $L_{n-1}^{J}$ is associated Laguerre polynomials, $n$ is radial quantum number, and $J$ is angular momentum quantum number; $C_{1}=0$ corresponds to $K<0$ and $C_{2}=0$ corresponds to $K>0$. The energy spectrum and radius parameter is given by

$$
\begin{gathered}
N w=\frac{\mathcal{E}}{\sqrt{1+\eta+\sqrt{(1+\eta)^{2}+8 \mathcal{E}}}}, \quad N=n+\frac{1}{2}(J-1), \\
r_{n}=2 \rho \sqrt{\frac{N}{\mathcal{E}}}=2 M \lambda \sqrt{\frac{N}{\mathcal{E}}} .
\end{gathered}
$$

Substituting (2.19) into (3.3) we get Regge-like relation as follows

$$
2 n+J-1=\frac{\sqrt{2}}{2 w}(1-2 \eta+M) \sqrt{2-\eta+M} .
$$

Or inversely,

$$
\begin{gathered}
M=\frac{1}{36} \mathcal{N}^{\frac{2}{3}}+4(1+\eta)^{2} \mathcal{N}^{-\frac{2}{3}}+\frac{1}{3}(5 \eta-4), \\
\mathcal{N}=216 N w \sqrt{2}+24 \sqrt{162 N^{2} w^{2}-3(1+\eta)^{3}} .
\end{gathered}
$$

In (3.5), we have 3 constants $(w, \eta, \mu)$ for the same series of particles to be determined by empirical data. Although the form of (3.5) or (3.6) is quite different from (1.1), the following calculation shows that the curves of (3.5) in the effective domain are quite near straight lines (see Figure 1).

Substituting (3.1) and (3.3) into (2.17), we can derive $V$. By calculation we get

$$
\int_{0}^{\infty} U_{K, n}^{2} r^{2} \mathrm{~d} r=\frac{(1+\eta) \sqrt{(1+\eta)^{2}+8 \mathcal{E}}+(1+\eta)^{2}+4 \mathcal{E}}{(1+\eta) \sqrt{(1+\eta)^{2}+8 \mathcal{E}}+(1+\eta)^{2}+6 \mathcal{E}} .
$$

For all meaningful eigen solutions, we have $0.1<\mathcal{E}<4$, and then we have $\int_{0}^{\infty} U_{K, n}^{2} r^{2} \mathrm{~d} r=0.8 \sim 1$. Therefore, the relative truncation error for the 0th approximation is about $10 \%$.

The ground state corresponds to $n=1, J=\frac{1}{2}$, and then we have $N=\frac{3}{4}$. Considering energy degeneracy, we only need to calculate the energy spectrums while $K \geq 1$. For the ground state of proton, we have empirical data 


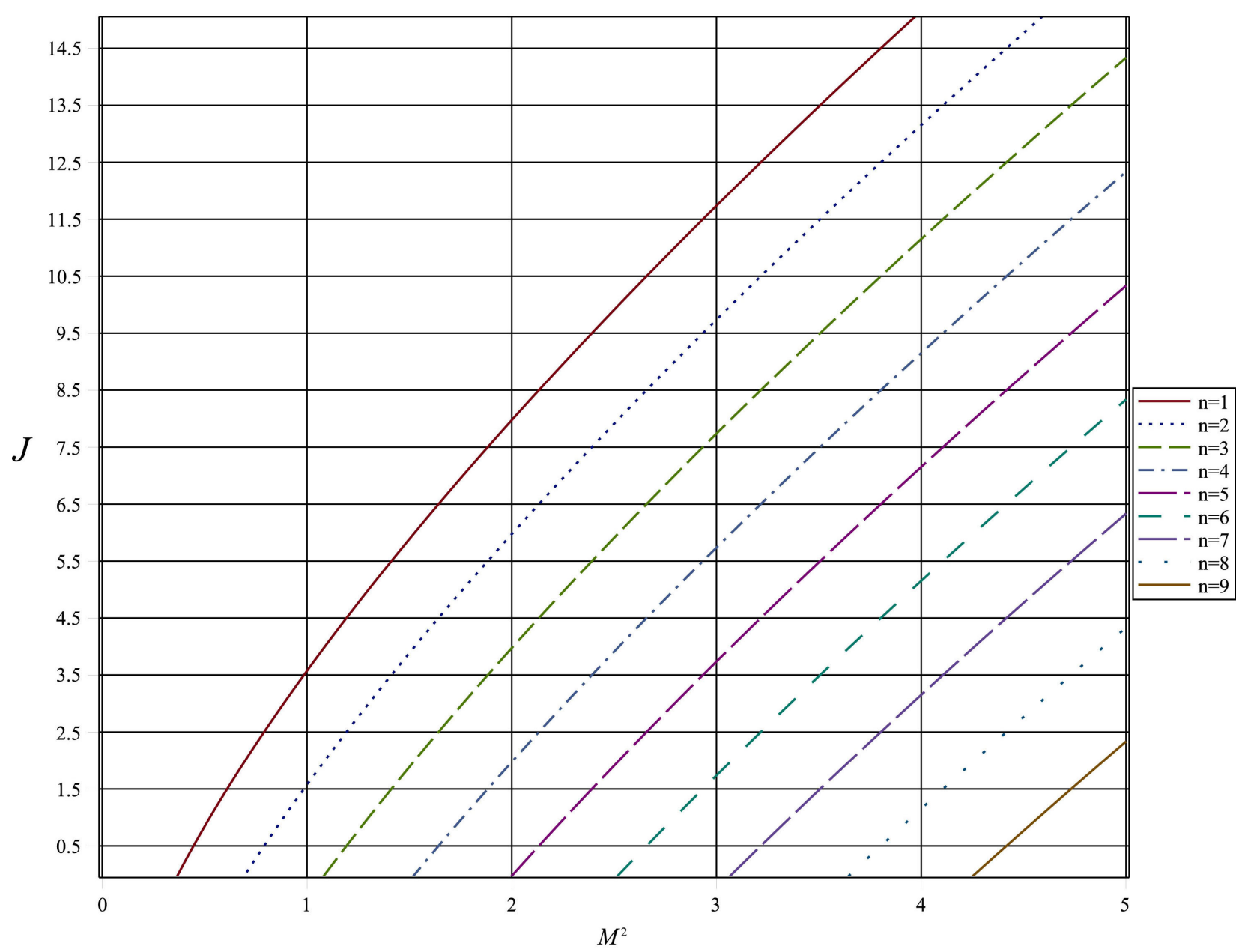

Figure 1. Regge trajectroies of (3.5). Each intersection between lines $J=\frac{1}{2}+j$ and curves $J=J\left(n, M^{2}\right)$ corresponds to one or more particles, and we have about 90 intersections in the figure. Considering degenerate states, the figure contains more than 1000 particles with different quantum numbers $\left(n, K, m_{z}, s\right)$.

$r_{n} \sim 1 \times 10^{-15} \mathrm{~m}$ and $m_{p}=938.28 \mathrm{MeV}$. Substituting them and $N=\frac{3}{4}$ into (2.11), (2.12), (2.18) and (3.4), we get constants $\left(w, \eta, \rho, \mu, \varepsilon_{0}\right)$ expressed by $\mathcal{E}_{0}$. If taking $\mathcal{E}_{0}=0.17618$, we have solution

$$
\begin{gathered}
\eta=0.74238, w=0.11972, \varepsilon_{0}=0.33220, \\
\rho=1.4048 \times 10^{-16} \mathrm{~m}, \mu c^{2}=1.4051 \mathrm{GeV} .
\end{gathered}
$$

For a proton, by (3.9) we find $r_{n} \sim 7 \rho$. By (2.11) and $\varepsilon_{0}$ we find the relative mass defect of strong interaction confinement is about $33 \%$. The observational mass $m_{p}$ is much less than constant mass $\mu$. This case is quite different from an electron without strong interaction.

Substituting (3.9) and $(n, K)$ into (3.5), (3.6) and (3.7), we find the masses of many baryons are near the spectra, and (3.5) is quite similar to the Regge trajectories of baryons (see Figure 1). By (3.3) and (3.4), we find the radius parameter $r_{n}$ of the excited states even decreases a little when the quantum number $N$ in- 
creases. The detailed calculation shows we always have $\bar{r}=(4 \sim 10) \rho$ for all particles. This means a particle with local parabolic potential or short distance potential $\Phi_{\alpha}$ has a very hard core. This phenomenon is quite different from the case of Coulomb potential, where we have $\bar{r} \propto n^{2}$.

For convenience, we take $J$ as row index and $n$ as column index, then the mass spectrums of the eigen states are listed in Table 1.

We find the masses of many baryons are near the spectra. Obviously each excited state should correspond to an observable particle. This means some baryons can be regarded as excited resonances of a proton. How to exactly identify the quantum numbers for each particle observed in experiments is an important but fallible problem.

As the 0th order approximation with only 3 free coefficients, the result is satisfactory. To get more accurate solutions of (1.2), we can expand $\phi$ as series of the eigen functions of (2.9) and then solve mass spectra of (1.2) [60]. However, in this case, we have only numerical results without an overview on the spectra.

\section{Effectiveness of the Parabolic Potential}

Now we check the effectiveness of the parabolic potential for nuclear potential. It is well known the global parabolic potential cannot be used as confining potential of Dirac equation. However, the following calculations show the local parabolic potential is effective to describe nuclear potential approximately.

At first, we check all radial functions $\left(U_{K, n}, V_{K, n}\right)$ and their module $U^{2}+V^{2}$ are almost distributed in the domain $r<12$, where is also the effective area of the local parabolic potential. The first couple of the radial functions is given by

$$
U_{1,1}=0.29450687 \mathrm{e}^{-0.05873333 r^{2}}, \quad V_{1,1}=-0.01796750 r \mathrm{e}^{-0.05873333 r^{2}},
$$

we find $|V / U|^{2} \sim 1 \%$. See Figure 2 and Figure 3 as follows, where we take $\rho=1$

Table 1. Mass spectra of Dirac equation with local parabolic potential (MeV).

\begin{tabular}{|c|c|c|c|c|c|c|c|c|c|c|c|c|c|c|}
\hline$m(J, n)$ & 1 & 2 & 3 & 4 & 5 & 6 & 7 & 8 & 9 & 10 & 11 & 12 & 13 & 14 \\
\hline $1 / 2$ & 938 & 1249 & 1535 & 1801 & 2052 & 2291 & 2520 & 2740 & 2953 & 3159 & 3359 & 3554 & 3744 & 3930 \\
\hline $1+1 / 2$ & 1098 & 1395 & 1670 & 1928 & 2173 & 2407 & 2631 & 2847 & 3056 & 3260 & 3457 & 3650 & 3838 & 4022 \\
\hline $2+1 / 2$ & 1249 & 1535 & 1801 & 2052 & 2291 & 2520 & 2740 & 2953 & 3159 & 3359 & 3554 & 3744 & 3930 & 4112 \\
\hline $3+1 / 2$ & 1395 & 1670 & 1928 & 2173 & 2407 & 2631 & 2847 & 3056 & 3260 & 3457 & 3650 & 3838 & 4022 & 4202 \\
\hline $4+1 / 2$ & 1535 & 1801 & 2052 & 2291 & 2520 & 2740 & 2953 & 3159 & 3359 & 3554 & 3744 & 3930 & 4112 & 4290 \\
\hline $5+1 / 2$ & 1670 & 1928 & 2173 & 2407 & 2631 & 2847 & 3056 & 3260 & 3457 & 3650 & 3838 & 4022 & 4202 & 4378 \\
\hline $6+1 / 2$ & 1801 & 2052 & 2291 & 2520 & 2740 & 2953 & 3159 & 3359 & 3554 & 3744 & 3930 & 4112 & 4290 & 4465 \\
\hline $7+1 / 2$ & 1928 & 2173 & 2407 & 2631 & 2847 & 3056 & 3260 & 3457 & 3650 & 3838 & 4022 & 4202 & 4378 & 4552 \\
\hline $8+1 / 2$ & 2052 & 2291 & 2520 & 2740 & 2953 & 3159 & 3359 & 3554 & 3744 & 3930 & 4112 & 4290 & 4465 & 4637 \\
\hline $9+1 / 2$ & 2173 & 2407 & 2631 & 2847 & 3056 & 3260 & 3457 & 3650 & 3838 & 4022 & 4202 & 4378 & 4552 & 4722 \\
\hline $10+1 / 2$ & 2291 & 2520 & 2740 & 2953 & 3159 & 3359 & 3554 & 3744 & 3930 & 4112 & 4290 & 4465 & 4637 & 4806 \\
\hline $11+1 / 2$ & 2407 & 2631 & 2847 & 3056 & 3260 & 3457 & 3650 & 3838 & 4022 & 4202 & 4378 & 4552 & 4722 & 4889 \\
\hline
\end{tabular}




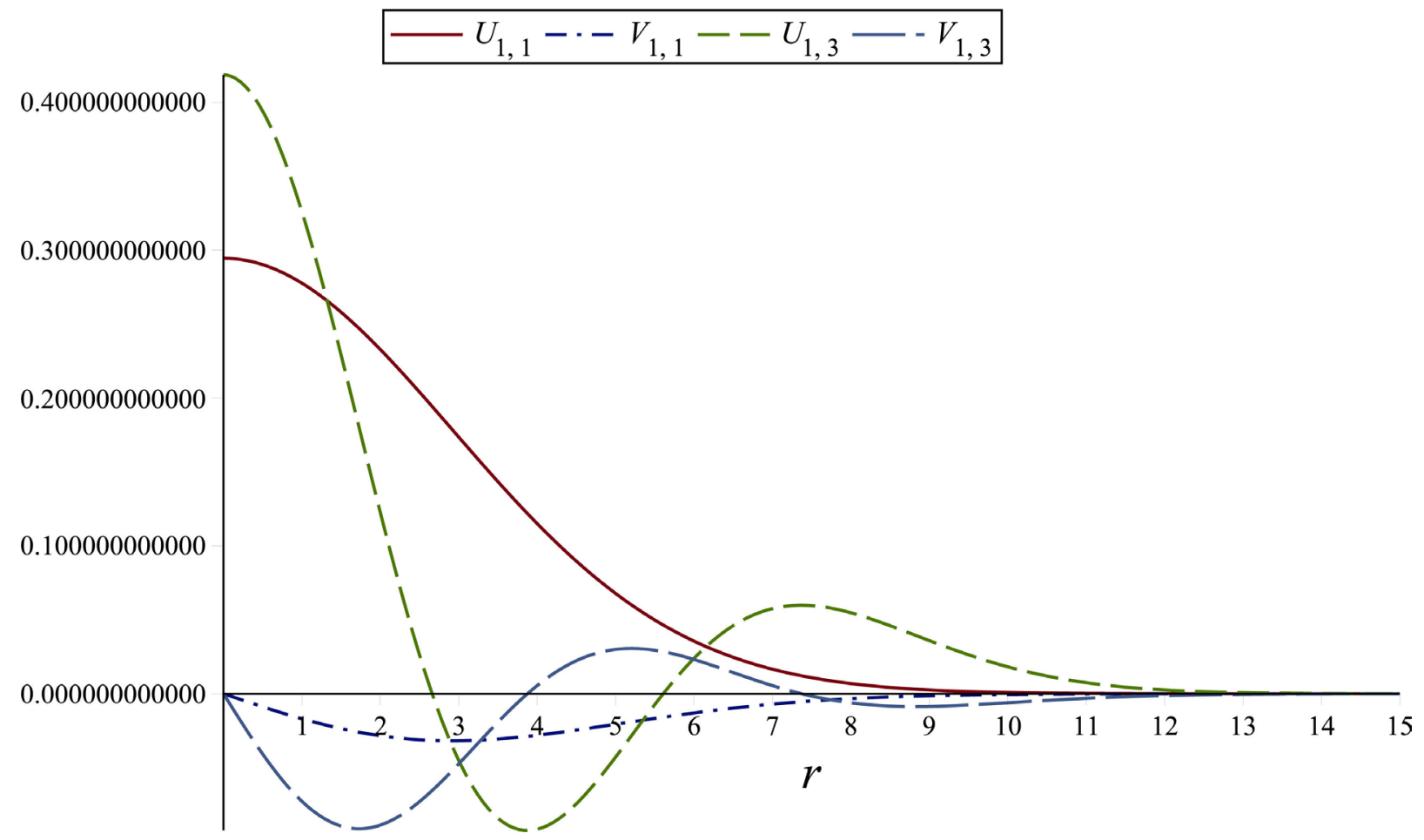

Figure 2. Some radial wave functions of a spinor in local parabolic potential.

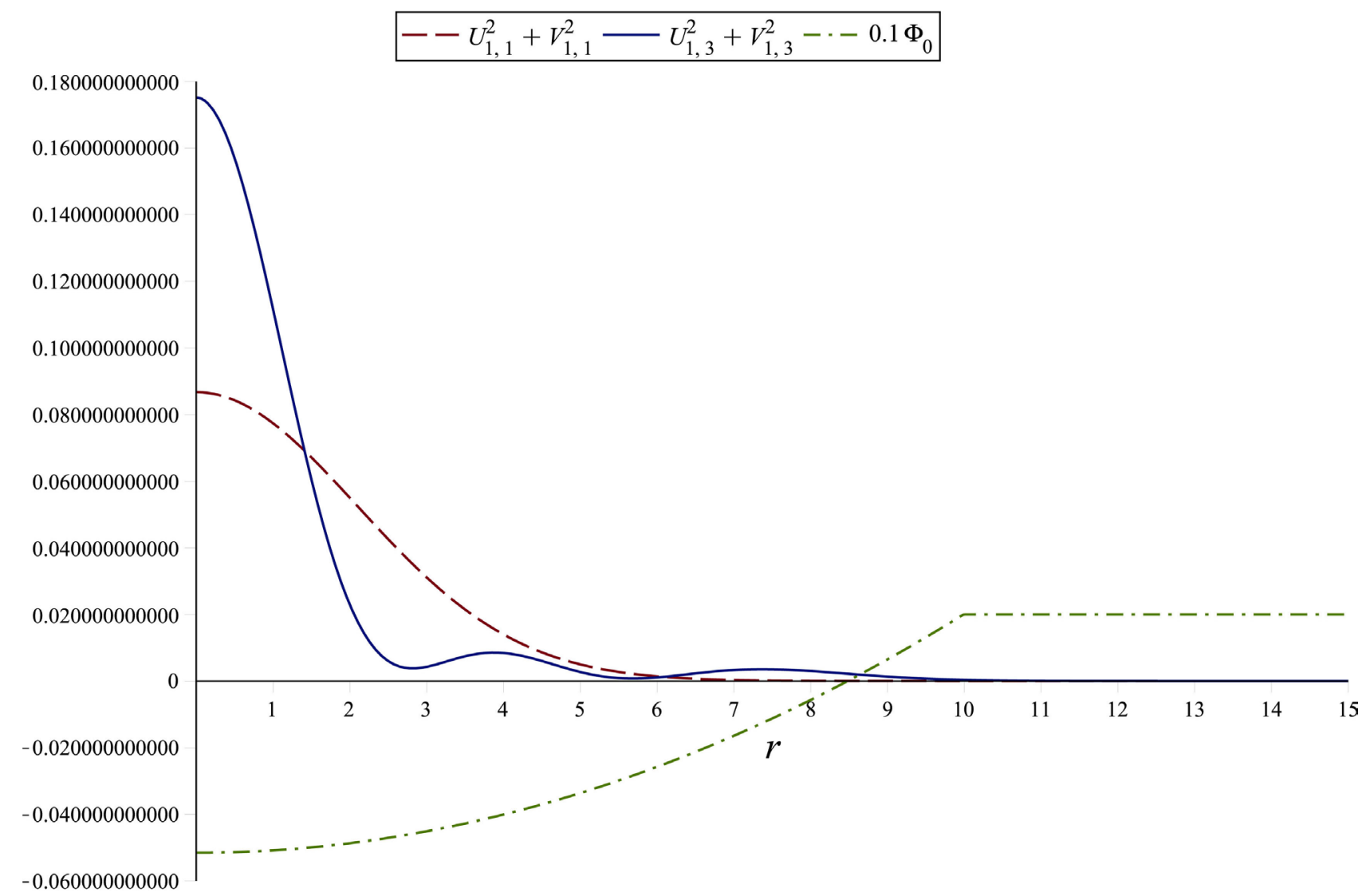

Figure 3. The effective domain of local parabolic potential and modules of radial wave functions. The spinor with short distance potential is mainly concentrated near the center, and it does not diffuse when $K$ or $n$ increases. 
as length unit.

Secondly, for the following short-range potential $\Phi$ with source $q(r)$,

$$
\partial_{\alpha} \partial^{\alpha} \Phi+\frac{1}{\rho^{2}} \Phi=-65 \cdot \frac{4 \pi q(r)}{\rho^{3}} .
$$

By Figure 4, we find the solution $\Phi$ is almost parabolic potential $\Phi_{0}$ in the domain $r<8$ for the above source, for which the normalizing condition is $4 \pi \int q(r) r^{2} \mathrm{~d} r=1$. This means in the interior of a baryon, the potential of strong interaction may be different from the Cornell potential or potential generated by point source $-\frac{\mathrm{e}^{-r}}{r}$ or the MIT bag model. It may be more suitably described by local parabolic potential.

Thirdly, by (3.3) we find that, different from electron in Coulomb potential, in this case, the radius parameter $r_{n}$ of the wave function even decreases a little as the increasing of quantum number $N$. So the local parabolic potential is also suitable for the excited states.

\section{Discussion and Conclusion}

As the 0th order approximation, the above calculation provides some important messages. The Dirac equation with short distance potential has quite different energy spectrum and eigen functions from that with Coulomb potential. Dirac equation is a magic equation with marvellous properties which should be strictly analyzed [61] [62] [63]. The nuclear potential may be more similar to local parabolic potential, rather than the MIT bag model or $-\frac{\mathrm{e}^{-r}}{r}$ or $-\frac{\alpha_{s}}{r}-a r$. Obviously,

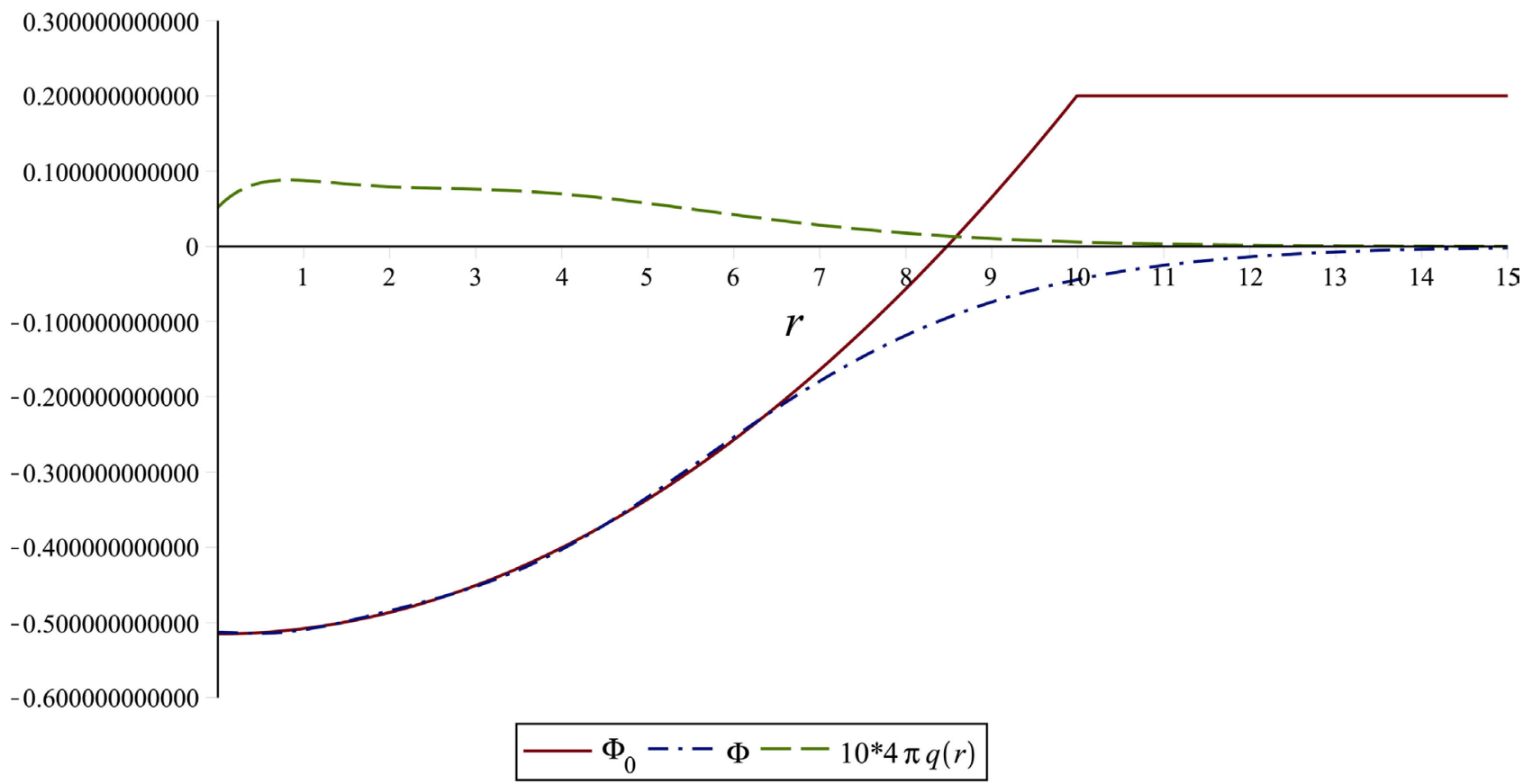

Figure 4. The parabolic potential versus the short range potential generated by $q(r)$. 
the spinor in short distance potential (4.2) has the asymptotic freedom near the center and strong confinement near $r \sim 10 \rho$ (see Figure 3 and Figure 4).

To get more accurate results, we should directly calculate the coupling system of (2.4) and (2.3), and expand the radial functions $(U, V)$ upon the bases $U_{K, n}, V_{K, n}$ of the representation space [60]. However, in this case, we have not explicit analytic expression (3.3) for mass spectra.

As the alternative models for fundamental particles, some simple and closed systems such as the following one are worth to be carefully studied,

$$
\begin{aligned}
\mathcal{L}= & \phi^{+} \alpha^{\mu}\left(i \partial_{\mu}-e A_{\mu}-s \Phi_{\mu}\right) \phi-\mu c \breve{\gamma}+F(\breve{\gamma}, \breve{\beta}) \\
& -\frac{1}{2} \partial_{\mu} A_{\alpha} \partial^{\mu} A^{\alpha}+\frac{1}{2}\left(\partial_{\mu} \Phi_{\alpha} \partial^{\mu} \Phi^{\alpha}-b^{2} \Phi_{\mu} \Phi^{\mu}\right) .
\end{aligned}
$$

Some deep secrets may be concealed under the nonlinear potential $F$ and short distance potentials, because the spinor equation is a magic equation.

If we denote $\phi \doteq \psi_{-1}+\psi_{0}+\psi_{1}$, where $\psi_{k}$ are basis eigenfunctions in the Hilbert space of representation, and $\psi_{0}$ is the main component. Substituting them into (5.1) and using the orthogonality of $\psi_{k}$, we get

$$
\begin{aligned}
\mathcal{L} \doteq & \sum_{k=-1}^{1}\left(\psi_{k}^{+} \alpha^{\mu}\left(i \partial_{\mu}-e A_{\mu}-s \Phi_{\mu}\right) \psi_{k}-\mu c \breve{\gamma}_{k}+F\left(\breve{\gamma}_{k}, \breve{\beta}_{k}\right)\right) \\
& -\frac{1}{2} \partial_{\mu} A_{\alpha} \partial^{\mu} A^{\alpha}+\frac{1}{2}\left(\partial_{\mu} \Phi_{\alpha} \partial^{\mu} \Phi^{\alpha}-b^{2} \Phi_{\mu} \Phi^{\mu}\right)+G\left(\psi_{k}, A^{\alpha}, \Phi^{\alpha}\right) .
\end{aligned}
$$

In (5.2), $\left(\psi_{-1}, \psi_{0}, \psi_{1}\right)$ may be easily interpreted as quarks with fraction electric charge and confinement, and the cross terms $G$ may be interpreted as gauge fields. For any complicated mathematical models, a little vigilance should be remained, because Nature only uses simple but best mathematics, and the complicated equations easily lead to inconsistence and singularity.

On the other hand, the regression analysis for empirical data to derive mass function with single integer variable $m=m(N),(N=1,2,3, \cdots)$ for similar particles is much important, because like Hydrogen spectra $E_{N}=\frac{1}{2} \hbar \omega \alpha^{2} N^{-2}$, such analytic function certainly exists and is usually very simple, and then to determine the further relation between quantum numbers $N=a n+b J+m_{0}$ is relatively easy. This procedure needs not to concern the physical meanings of $N$ at first and gets rid of the fallible and misleading task to identify the quantum numbers $n$ and $J$ for each particle at the beginning. If we can arrange the masses of similar particles from small to large at each horizontal integer coordinates $N$ to get smooth curves, the regressive function $m=m(N)$ for all smooth curves can be derived. From the final mass function $m=m\left(a n+b J+m_{0}\right)$ of high precision, we can determine the specific potentials in Dirac equation conversely.

\section{Conflicts of Interest}

The authors declare no conflicts of interest regarding the publication of this paper. 


\section{References}

[1] Chew, G.F. and Frautschi, S.C. (1962) Regge Trajectories and the Principle of Maximum Strength for Strong Interactions. Physical Review Letters, 8, 41-44.

https://doi.org/10.1103/PhysRevLett.8.41

[2] Frautschi, S.C. (1968) Regge Poles and S-Matrix Theory. W. A. Benjamin, New York.

[3] Afonin, S.S. (2006) Towards Understanding Spectral Degeneracies in Nonstrange Hadrons. Part I: Mesons as Hadron Strings vs. Phenomenology.

[4] Allen, T.J., Goebel, C., Olsson, M.G. and Veseli, S. (2001) Analytic Quantization of the QCD String. Physical Review D, 64, Article ID: 094011. https://doi.org/10.1103/PhysRevD.64.094011

[5] Isgur, N. and Karl, G. (1978) P-Wave Baryons in the Quark Model. Physical Review $D, 18,4187$. https://doi.org/10.1103/PhysRevD.18.4187

[6] Copley, L.A., Isgur, N. and Karl, G. (1979) Charmed Baryons in a Quark Model with Hyperfine Interactions, Physical Review D, 20, 768. https://doi.org/10.1103/PhysRevD.20.768

[7] Isgur, N. (1981) Isospin-Violating Mass Differences and Mixing Angles: The Role of Quark Masses. Physical Review D, 23, 817. https://doi.org/10.1103/PhysRevD.23.817.4

[8] Yoshida, T., Hiyama, E., Hosaka, A., Oka, M. and Sadato, K. (2015) Spectrum of Heavy Baryons in the Quark Model. Physical Review D, 92, Article ID: 114029. https://doi.org/10.1103/PhysRevD.92.114029

[9] Rai, A.K., Patel, B. and Vinodkumar, P.C. (2008) Properties of QQ Mesons in Non-Relativistic QCD Formalism. Physical Review C, 78, Article ID: 055202. https://doi.org/10.1103/PhysRevC.78.055202

[10] Roberts, W. and Pervin, M. (2008) Heavy Baryons in a Quark Model. International Journal of Modern Physics A, 23, 2817-2860. https://doi.org/10.1142/S0217751X08041219

[11] Capstick, S. and Isgur, N. (1986) Baryons in a Relativized Quark Model with Chromodynamics. Physical Review D, 34, 2809. https://doi.org/10.1103/PhysRevD.34.2809

[12] Melde, T., et al. (2008) Quark-Model Identification of Baryon Ground and Resonant States. Physical Review D, 77, Article ID: 114002. https://doi.org/10.1143/JPSJ.77.114002

[13] Thakkara, K., Shahb, Z., Raib, A.K. and Vinodkumar, P.C. (2017) Excited State Mass Spectra and Regge Trajectories of Bottom Baryons. Nuclear Physics A, 965, 57-73. https://doi.org/10.1016/j.nuclphysa.2017.05.087

[14] Nambu, Y. (1974) Strings, Monopoles and Gauge Fields. Physical Review D, 10, 4262. https://doi.org/10.1103/PhysRevD.10.4262

[15] Isgur, N. and Paton, J. (1985) Flux-Tube Model for Hadrons in QCD. Physical Review $D$, 31, 2910-2929. https://doi.org/10.1103/PhysRevD.31.2910

[16] Baker, M. and Steinke, R. (2002) Semiclassical Quantization of Effective String Theory and Regge Trajectories. Physical Review D, 65, Article ID: 094042. https://doi.org/10.1103/PhysRevD.65.094042

[17] Baker, M. and Steinke, R. (2001) Effective String Theory of Vortices and Regge Trajectories. Physical Review D, 63, Article ID: 094013. https://doi.org/10.1103/PhysRevD.63.094013 
[18] LaCourse, D. and Olsson, M.G. (1989) The String Potential Model. 1. Spinless Quarks. Physical Review D, 39, 2751. https://doi.org/10.1103/PhysRevD.39.2751

[19] Chen, B., Wei, K.W. and Zhang, A. (2015) Assignments of $\Lambda_{\mathrm{Q}}$ and $\Xi_{\mathrm{Q}}$ Baryons in the Heavy Quark-Light Diquark Picture. The European Physical Journal A, 51, 82. https://doi.org/10.1140/epja/i2015-15082-3

[20] Sharov, G.S. (2000) Quasirotational Motions and Stability Problem in Dynamics of String Hadron Models. Physical Review D, 62, Article ID: 094015. https://doi.org/10.1103/PhysRevD.62.094015

[21] Chen, K., Dong, Y.B., Liu, X., Lü, Q.F. and Matsuki, T. (2018) Regge-Like Relation and a Universal Description of Heavy-Light Systems. The European Physical Journal $C, \mathbf{7 8}, 20$.

[22] Afonin, S.S. (2007) Properties of New Unflavored Mesons below $2.4 \mathrm{GeV}$. Physical Review C, 76, Article ID: 015202. https://doi.org/10.1103/PhysRevC.76.015202

[23] Matsuki, T. and Morii, T. (1997) Spectroscopy of Heavy Mesons Expanded in 1/mQ. Physical Review D, 56, 5646. https://doi.org/10.1103/PhysRevD.56.5646

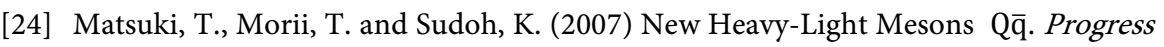
of Theoretical Physics, 117, 1077. https://doi.org/10.1143/PTP.117.1077

[25] Veseli, S. and Olsson, M.G. (1996) Sum Rules, Regge Trajectories, and Relativistic Quark Models. Physics Letters B, 383, 109. https://doi.org/10.1016/0370-2693(96)00721-6

[26] Matsuki, T., Lü, Q.F., Dong, Y. and Morii, T. (2016) Approximate Degeneracy of Heavy-Light Mesons with the Same L. Physics Letters B, 758, 274. https://doi.org/10.1016/j.physletb.2016.05.016

[27] Ebert, D., Faustov, R.N. and Galkin, V.O. (2011) Spectroscopy and Regge Trajectories of Heavy Baryons in the Relativistic Quark-Diquark Picture. Physical Review D, 84, Article ID: 014025. https://doi.org/10.1103/PhysRevD.84.014025

[28] Page, P.R., Goldman, J.T. and Ginocchio, J.N. (2001) Relativistic Symmetry Suppresses Quark Spin-Orbit Splitting. Physical Review Letters, 86, 204. https://doi.org/10.1103/PhysRevLett.86.204

[29] Riazuddin and Shafiq, S. (2012) Spin-Orbit Splittings in Heavy-Light Mesons and Dirac Equation. The European Physical Journal C, 72, 1925. https://doi.org/10.1140/epjc/s10052-012-1925-1

[30] Jia, D., Pang, C.Q. and Hosaka, A. (2017) Mass Formula for Light Nonstrange Mesons and Regge Trajectories in Quark Model. International Journal of Modern Physics A, 32, Article ID: 1750153. https://doi.org/10.1142/S0217751X17501536

[31] Ebert, D., Faustov, R.N. and Galkin, V.O. (2009) Mass Spectra and Regge Trajectories of Light Mesons in the Relativistic Quark Model. Physical Review D, 79, Article ID: 114029. https://doi.org/10.1103/PhysRevD.79.114029

[32] Yamaguchi, Y., Ohkoda, S., Hosaka, A., Hyodo, T. and Yasui, S. (2015) Heavy Quark Symmetry in Multihadron Systems, Physical Review D, 91, Article ID: 034034. https://doi.org/10.1103/PhysRevD.91.034034

[33] Mao, Q., Chen, H.X., Chen, W., Hosaka, A., et al. (2015) QCD Sum Rule Calculation for P-Wave Bottom Baryons. Physical Review D, 92, Article ID: 114007. https://doi.org/10.1103/PhysRevD.92.114007

[34] Liu, X., Chen, H.X., Liu, Y.R., Hosaka, A. and Zhu, S.L. (2008) Bottom Baryons. Physical Review D, 77, Article ID: 014031. https://doi.org/10.1103/PhysRevD.77.014031

[35] Patel, B., Rai, A.K. and Vinodkumar, P.C. (2008) Pramana, Heavy Flavour Baryons 
in Hyper Central Model. Journal of Physics, 70, 797. https://doi.org/10.1007/s12043-008-0090-4

[36] Karliner, M. and Rosner, J.L. (2015) Prospects for Observing the Lowest-Lying Odd-Parity $\Sigma_{\mathrm{c}}$ and $\Sigma_{\mathrm{b}}$ Baryons. Physical Review D, 92, Article ID: 074026. https://doi.org/10.1103/PhysRevD.92.074026

[37] Karliner, M., Keren-Zur, B., Lipkin, H.J. and Rosner, J.L. (2009) The Quark Model and b Baryons. Annals of Physics (Amsterdam), 324, 2-15. https://doi.org/10.1016/j.aop.2008.05.003

[38] Llanes-Estrada, F.J., Cotanch, S.R., Szczepaniak, A.P. and Swanson, E.S. (2004) Hyperfine Meson Splittings: Chiral Symmetry versus Transverse Gluon Exchange. Physical Review C, 70, Article ID: 035202. https://doi.org/10.1103/PhysRevC.70.035202

[39] Thakkar, K., Majethiya, A. and Vinodlumar, P.C. (2016) Magnetic Moments of Baryons Containing All Heavy Quarks in Quark-Diquark Model. The European Physical Journal Plus, 131, 339. https://doi.org/10.1140/epjp/i2016-16339-4

[40] Fodor, Z. and Hoelbling, C. (2012) Light Hadron Masses from Lattice QCD. Reviews of Modern Physics, 84, 449. https://doi.org/10.1103/RevModPhys.84.449

[41] Brown, Z.S., Detmold, W., Meinel, S. and Orginos, K. (2014) Charmed Bottom Baryon Spectroscopy from Lattice QCD. Physical Review D, 90, Article ID: 094507. https://doi.org/10.1103/PhysRevD.90.094507

[42] Padmanath, M. and Mathur, N. (2017) Quantum Numbers of Recently Discovered $\pi_{c}^{0}$ Baryons from Lattice QCD. Physical Review Letters, 119, Article ID: 042001. https://doi.org/10.1103/PhysRevLett.119.042001

[43] Lewis, R. and Woloshyn, R.M. (2009) Bottom Baryons from a Dynamical Lattice QCD Simulation. Physical Review D, 79, Article ID: 014502. https://doi.org/10.1103/PhysRevD.79.014502

[44] Tang, A. and Norbury, J.W. (2000) Properties of Regge Trajectories. Physical Review D, 62, 598-603. https://doi.org/10.1103/PhysRevD.62.016006

[45] Inopin, A.E. (2001) Hadronic Regge Trajectories in the Resonance Energy Region.

[46] Masjuan, P. and Arriola, E.R. (2017) Regge Trajectories of Excited Baryons, QuarkDiquark Models and Quark-Hadron Duality. Physical Review D, 96, Article ID: 054006.

[47] Bisht, S., Hothi, N. and Bhakuni, G. (2010) Phenomenological Analysis of Hadronic Regge Trajectories. Electronic Journal of Theoretical Physics, 7, 299-318.

[48] Wei, K.W., Chen, B., Liu, N., Wang, Q.Q. and Guo, X.H. (2017) Spectroscopy of Singly, Doubly, and Triply Bottom Baryons. Physical Review D, 95, Article ID: 116005. https://doi.org/10.1103/PhysRevD.95.116005

[49] Brau, F. (2000) Bohr-Sommerfeld Quantization and Meson Spectroscopy, Physical Review D, 62, Article ID: 014005. https://doi.org/10.1103/PhysRevD.62.014005

[50] Olive, K.A., et al. (2014) Review of Particle Physics. Chinese Physics C, 38, Article ID: 090001. https://doi.org/10.1088/1674-1137/38/9/090001

[51] Aaij, R., et al. (2017) Observation of Five New Narrow $\pi_{c}^{0}$ States Decaying to $\Xi_{\mathrm{c}}^{+} \mathrm{k}^{-}$. Physical Review Letters, 118, Article ID: 182001.

[52] Aaij, R., et al. (2016) Observation of $\Lambda_{b}{ }^{0} \rightarrow \psi(2 S) p K^{-}$and $\Lambda_{b}{ }^{0} \rightarrow J / \psi \pi^{+} \pi^{-} p K^{-}$ Decays and a Measurement of the $\Lambda_{b}{ }^{0}$ Baryon Mass. Journal of High Energy Physics, 1605, 132.https://doi.org/10.1007/JHEP05(2016)132 
[53] Aaij, R., et al. (2012) Observation of Excited $\Lambda_{b}{ }^{0}$ Baryons. Physical Review Letters, 109, Article ID: 172003. https://doi.org/10.1103/PhysRevLett.109.172003

[54] Chatrchyan, S., et al. (2012) Observation of a New $\Xi b$ Baryon. Physical Review Letters, 108, Article ID: 252002. https://doi.org/10.1103/PhysRevLett.108.252002

[55] Patrignani, C., et al. (2016) Review of Particle Physics. Chinese Physics C, 40, Article ID: 100001. https://doi.org/10.1088/1674-1137/40/10/100001

[56] Hagiwara, K., et al. (2002) Review of Particle Properties. Physical Review D, 66, Article ID: 010001. https://doi.org/10.1103/PhysRevD.66.010001

[57] Caso, C., et al. (1998) Review of Particle Physics. The European Physical Journal C, 3, 1. https://doi.org/10.1007/s10052-998-0104-X

[58] Crede, V. and Roberts, W. (2013) Progress toward Understanding Baryon Resonances. Reports on Progress in Physics, 76, Article ID: 076301. https://doi.org/10.1088/0034-4885/76/7/076301

[59] Gu, Y.Q. (2008) Integrable Conditions for Dirac Equation and Schrödinger Equation.

[60] Gu, Y.Q. (2017) A Procedure to Solve the Eigen Solution to Dirac Equation. Quantum Physics Letters, 6, 161-163.

[61] Gu, Y.Q. (2007) New Approach to N-Body Relativistic Quantum Mechanics. International Journal of Modern Physics A, 22, 2007-2020. https://doi.org/10.1142/S0217751X07036233

[62] Gu, Y.Q. (2018) Clifford Algebra, Lorentz Transformation and Unified Field Theory. Advances in Applied Clifford Algebras, 28, 37. https://doi.org/10.1007/s00006-018-0852-0

[63] Gu, Y.Q. (2018) Space-Time Geometry and Some Applications of Clifford Algebra in Physics. Advances in Applied Clifford Algebras, 28, 79. https://doi.org/10.1007/s00006-018-0896-1 